\title{
Nonexistence of self-similar singularities in the ideal magnetohydrodynamics
}

\author{
Dongho Chae* \\ Department of Mathematics \\ Sungkyunkwan University \\ Suwon 440-746, Korea \\ e-mail : chae@skku.edu
}

\begin{abstract}
In this paper we exclude the scenario of apparition of finite time singularity in the form of self-similar singularities in the ideal magnetohydrodynamic equations, assuming suitable integrability conditions on the vorticity and the magnetic field. We also consider more sophisticated possibility of asymptotically self-similar singularities, which means that the local classical solution converges to the self-similar profile as we approaches to the possible time of singularity. The scenario of asymptotically self-similar singularity is also excluded under suitable conditions on the profile. In the 2D magnetohydrodynamics the magnetic field evolution equations reduce to a divergence free transport equation for a scalar stream function. This helps us to improve the above nonexistence theorems on the self-similar singularities, in the sense that we only need weaker integrability conditions on the profile to prove the results.
\end{abstract}

*This work was supported partially by KRF Grant(MOEHRD, Basic Research Promotion Fund) and the KOSEF Grant no. R01-2005-000-10077-0. Part of the work was done, while the author was visiting RIMS, Kyoto University. He would like to thank Professor Hisashi Okamoto for his hospitality during the visit and useful discussions.

Key Words: ideal magnetohydrodynamics, self-similar singularities, asymptotically selfsimilar singularities 


\section{Introduction}

We are concerned on the possibility of finite time singularity in the Cauchy problem of the ideal magnetohydrodynamic equations in $\mathbb{R}^{n}, n=2,3$.

$$
(\mathrm{MHD})\left\{\begin{array}{l}
\frac{\partial v}{\partial t}+(v \cdot \nabla) v=(b \cdot \nabla) b-\nabla\left(p+\frac{1}{2}|b|^{2}\right) \\
\frac{\partial b}{\partial t}+(v \cdot \nabla) b=(b \cdot \nabla) v \\
\quad \operatorname{div} v=\operatorname{div} b=0 \\
v(x, 0)=v_{0}(x), \quad b(x, 0)=b_{0}(x)
\end{array}\right.
$$

where $v=\left(v_{1}, \cdots, v_{n}\right), v_{j}=v_{j}(x, t), j=1, \cdots, n$, is the velocity of the flow, $p=p(x, t)$ is the scalar pressure, $b=\left(b_{1}, \cdots, b_{n}\right), b_{j}=b_{j}(x, t)$, is the magnetic field, and $v_{0}, b_{0}$ are the given initial velocity and magnetic field, satisfying $\operatorname{div} v_{0}=\operatorname{div} b_{0}=0$, respectively. The system (MHD) is the incompressible Euler equations coupled with the magnetic field evolution equations, and the question of finite time singularity/global regularity is an outstanding open problem in the mathematical fluid mechanics, similarly to the case of the 3D Euler equations(see [6], and references therein). There are some numerical approaches to this problem(see e.g. [14, 12] and references therein). The blow-up criterion similar to the Beale-Kato-Majda's one for incompressible Euler system([1]) is obtained by Caflisch, Klapper and Steele([3], see also [21]). In this paper our aim is to consider the possibility of finite time apparition of singularity for (MHD) in the form of self-similar type, and exclude it. The organization of this paper is the following:

In the section 1 we first exclude self-similar singularities of the Leray type for the Navier-Stokes equations introduced in [18]. In the section 2, using the result of section 1 , we exclude more sophisticated self-similar singularity, called the asymptotically self-similar singularities. In the section 3 we present improved theorems of sections 1 and 2 in the case of planar magnetohydrodynamics.

\section{Self-similar singularities}

In order to be more precise on the notion of self-similar singularities we begin by the following observation on the scaling property of (MHD): if $(v, b, p)$ is a solution of (MHD) corresponding to the initial data $\left(v_{0}, b_{0}\right)$, then for any 
$\lambda>0$ and $\alpha \in \mathbb{R}$ the functions

$$
v^{\lambda, \alpha}(x, t)=\lambda^{\alpha} v\left(\lambda x, \lambda^{\alpha+1} t\right), \quad b^{\lambda, \alpha}(x, t)=\lambda^{\alpha} b\left(\lambda x, \lambda^{\alpha+1} t\right)
$$

and

$$
p^{\lambda, \alpha}(x, t)=\lambda^{2 \alpha} p\left(\lambda x, \lambda^{\alpha+1} t\right)
$$

are also solutions with the initial data $v_{0}^{\lambda, \alpha}(x)=\lambda^{\alpha} v_{0}(\lambda x), b_{0}^{\lambda, \alpha}(x)=\lambda^{\alpha} b_{0}(\lambda x)$. In view of the above scaling property the self-similar blowing up solution $(v(x, t), b(x, t))$ of the system (MHD) should be of the form,

$$
\begin{aligned}
& v(x, t)=\frac{1}{\left(T_{*}-t\right)^{\frac{\alpha}{\alpha+1}}} V\left(\frac{x}{\left(T_{*}-t\right)^{\frac{1}{\alpha+1}}}\right), \\
& b(x, t)=\frac{1}{\left(T_{*}-t\right)^{\frac{\alpha}{\alpha+1}}} B\left(\frac{x}{\left(T_{*}-t\right)^{\frac{1}{\alpha+1}}}\right), \\
& p(x, t)=\frac{1}{\left(T_{*}-t\right)^{\frac{2 \alpha}{\alpha+1}}} P\left(\frac{x}{\left(T_{*}-t\right)^{\frac{1}{\alpha+1}}}\right)
\end{aligned}
$$

for $\alpha \neq-1$ and $t$ close to the possible blow-up time $T_{*}$. If we substitute (1.1)-(1.3) into (MHD), then we find that $(V, B, P)$ should be a solution of the stationary system:

$$
\left\{\begin{array}{l}
\frac{\alpha}{\alpha+1} V+\frac{1}{\alpha+1}(y \cdot \nabla) V+(V \cdot \nabla) V=(B \cdot \nabla) B-\nabla\left(P+\frac{1}{2}|B|^{2}\right) \\
\frac{\alpha}{\alpha+1} B+\frac{1}{\alpha+1}(y \cdot \nabla) B+(V \cdot \nabla) B=(B \cdot \nabla) V \\
\quad \operatorname{div} V=\operatorname{div} B=0
\end{array}\right.
$$

Conversely, if $(V, B, P)$ is a smooth solution of the system (1.4), then the triple of functions $(v, b, p)$ defined by (1.1)-(1.3) is a smooth solution of (MHD) for $t \in\left(0, T_{*}\right)$, which blows up at $t=T_{*}$. The search for self-similar singularities of the form similar to (1.1)-(1.3) (more precisely with $\alpha=1$ and $B=b=0$ ) was suggested by Leray for the 3D Navier-Stokes equations in [18, and the possibility was first excluded by Nečas, Ružička and Šverák in [20] under the condition of $V \in L^{3}\left(\mathbb{R}^{3}\right) \cap H_{\text {loc }}^{1}\left(\mathbb{R}^{3}\right)$, the result of which was generalized later by Tsai in [23]. Their proofs crucially depends on the maximum principle of the Leray system,

$$
\frac{1}{2} V+\frac{1}{2}(y \cdot \nabla) V+(V \cdot \nabla) V=-\nabla P+\Delta V, \quad \operatorname{div} V=0,
$$


which corresponds to the Navier-Stokes version of the system (1.4). The maximum principle, in turn, is possible due to the dissipation term, $\Delta V$, in the Leray system, which is originated from the dissipation term of the Navier-Stokes equations. Due to this fact there was difficulty in extending the nonexistence results for the self-similar singularity of the 3D Navier-Stokes system to the 3D Euler equations, applying the similar method to [20] or [23]. Recently, the author of this paper discovered completely new argument to prove nonexistence of the self-similar singularity of the 3D Euler system under suitable integrability condition on the vorticity([4]). In this section we apply that method iteratively to prove nonexistence of self-similar singularity for (MHD). The precise theorems are stated and proved in the subsections below.

\section{$1.1 \quad$ Statement of the theorems}

Below we denote by $C_{0}^{m}\left(\mathbb{R}^{n}\right)$ the collection of $C^{m}\left(\mathbb{R}^{n}\right)$ functions with all the derivatives up to the $m$-th order vanishing at infinity. The class of $C^{m}\left(\mathbb{R}^{n}\right)$ functions with compact support will be denoted by $C_{c}^{m}\left(\mathbb{R}^{n}\right)$.

Theorem 1.1 Suppose there exists $T_{*}>0$ and $\alpha \neq-1$ such that we have a representation of a solution $(v, b)$ to $(M H D)$ by (1.1)-(1.2) for all $t \in\left(0, T_{*}\right)$ with $(V, B)$ satisfying the following conditions:

(i) $(V, B) \in\left[C_{0}^{1}\left(\mathbb{R}^{n}\right)\right]^{2}$.

(ii) There exists $q_{1}>0$ such that $(\Omega, B) \in\left[L^{q}\left(\mathbb{R}^{3}\right)\right]^{2}$ for all $q \in\left(0, q_{1}\right)$, where $\Omega=\operatorname{curl} V$.

Then, $V=B=0$.

Remark 1.1 Due to the condition (i) on $V$ we can exclude the possibility of the self-similar singular solution $(v, b, p)$ of the form,

$$
\begin{aligned}
v(x, t)= & \frac{1}{\left(T_{*}-t\right)^{\frac{\alpha}{\alpha+1}}} \nabla h\left(\frac{x}{\left(T_{*}-t\right)^{\frac{1}{\alpha+1}}}\right), \\
b(x, t)= & 0 \\
p(x, t)= & -\frac{1}{(\alpha+1)\left(T_{*}-t\right)^{\frac{\alpha+2}{\alpha+1}}}(x \cdot \nabla) h\left(\frac{x}{\left(T_{*}-t\right)^{\frac{1}{\alpha+1}}}\right) \\
& \quad-\frac{1}{2\left(T_{*}-t\right)^{\frac{2}{\alpha+1}}}\left|\nabla h\left(\frac{x}{\left(T_{*}-t\right)^{\frac{1}{\alpha+1}}}\right)\right|^{2}
\end{aligned}
$$


with a non-constant harmonic function $h$ and $\alpha \neq-1$, for which $V=\nabla h \notin$ $C_{0}^{1}\left(\mathbb{R}^{n}\right)$. Another reason for the condition (i) is that, since the local classical solution $v$ given by (1.1) satisfy $v(\cdot, t) \in C_{0}^{1}\left(\mathbb{R}^{n}\right)$ for $t \in\left(0, T_{*}\right)$, it guarantees the existence of back-to-label map generated by the velocity field $v$ (see [8]), which is importantly used in the proof(see the proof of Theorem 1.2 below).

Remark 1.2 In order to illustrate the decay condition (ii) we make the following observations. If $\Omega \in L^{\infty}\left(\mathbb{R}^{n}\right)$ and there exist constants $R, C, \varepsilon_{1}, \varepsilon_{2}>0$ such that $|\Omega(x)| \leq C e^{-\varepsilon_{1}|x|^{\varepsilon_{2}}}$ for $|x|>R$, then we have $\Omega \in L^{q}\left(\mathbb{R}^{n}\right)$ for all $q \in(0,1)$. Indeed, for all $q \in(0,1)$, we have

$$
\begin{aligned}
\int_{\mathbb{R}^{n}}|\Omega(x)|^{q} d x & =\int_{|x| \leq R}|\Omega(x)|^{q} d x+\int_{|x|>R}|\Omega(x)|^{q} d x \\
& \leq\left|B_{R}\right|^{1-q}\left(\int_{|x| \leq R}|\Omega(x)| d x\right)^{q}+C^{q} \int_{\mathbb{R}^{n}} e^{-q \varepsilon_{1}|x|^{\varepsilon_{2}}} d x<\infty
\end{aligned}
$$

where $\left|B_{R}\right|$ is the volume of $B_{R}$.

Theorem 1.1 will follow as a corollary of the following more general theorem.

Theorem 1.2 Suppose there exists $T>0$ such that we have a representation of the solution $(b, v)$ to $(M H D)$ by

$$
\begin{gathered}
\omega(x, t)=\Psi_{1}(t) \Omega\left(\Phi_{1}(t) x\right), \\
b(x, t)=\Psi_{2}(t) B\left(\Phi_{2}(t) x\right)
\end{gathered}
$$

for all $t \in[0, T)$, where $\omega=\operatorname{curl} v, \Omega=\operatorname{curl} V$ with $V$ satisfying div $V=0$, div $B=0, \Psi_{j}(\cdot) \in C([0, T) ;(0, \infty)), \Phi_{j}(\cdot) \in C\left([0, T) ; \mathbb{R}^{n \times n}\right)$ with $\operatorname{det}\left(\Phi_{j}(t)\right) \neq 0$ on $[0, T)$ for each $j=1,2$, and $(V, B)$ satisfying the conditions (i) and (ii) of Theorem 1.1. Then, necessarily either $\operatorname{det}\left(\Phi_{j}(t)\right) \equiv \operatorname{det}\left(\Phi_{j}(0)\right)$ on $[0, T)$ for $j=1,2$, or $V=B=0$.

\subsection{Proof of the theorems}

Proof of Theorem 1.2 We assume local classical solution $(v, b)$ of the form (1.7)-(1.8). We will show that this assumption leads to $\Omega=B=$ 
0 . By consistency with the initial condition, $b_{0}(x)=\Psi_{2}(0) B\left(\Phi_{2}(0) x\right)$, and hence $B(x)=\Psi_{2}(0)^{-1} b_{0}\left(\left[\Phi_{2}(0)\right]^{-1} x\right)$ for all $x \in \mathbb{R}^{n}$. We can rewrite the representation (1.8) in the form,

$$
b(x, t)=G(t) b_{0}(F(t) x) \quad \forall t \in[0, T),
$$

where $G(t)=\Psi_{2}(t) / \Psi_{2}(0), F(t)=\left[\Phi_{2}(0)\right]^{-1} \Phi_{2}(t)$. In order to prove the theorem it suffices to show that either $\operatorname{det}(F(t))=1$ for all $t \in[0, T)$, or $b_{0}=0$, since $\operatorname{det}(F(t))=\operatorname{det}\left(\Phi_{2}(t)\right) / \operatorname{det}\left(\Phi_{2}(0)\right)$. Let $a \mapsto X(a, t)$ be the particle trajectory mapping, defined by the ordinary differential equations,

$$
\frac{\partial X(a, t)}{\partial t}=v(X(a, t), t) \quad ; \quad X(a, 0)=a .
$$

We set $A(x, t):=X^{-1}(x, t)$, which is called the back-to-label map, satisfying

$$
A(X(a, t), t)=a, \quad X(A(x, t), t)=x .
$$

Taking dot product the second equation of (MHD) by $b$, we obtain

$$
\frac{\partial|b|}{\partial t}+(v \cdot \nabla)|b|=\alpha|b|
$$

where $\alpha(x, t)$ is defined as

$$
\alpha(x, t)=\left\{\begin{array}{cl}
\sum_{i, j=1}^{n} S_{i j}(x, t) \xi_{i}(x, t) \xi_{j}(x, t) & \text { if } \quad b(x, t) \neq 0 \\
0 & \text { if } \quad b(x, t)=0
\end{array}\right.
$$

with

$$
S_{i j}=\frac{1}{2}\left(\frac{\partial v_{j}}{\partial x_{i}}+\frac{\partial v_{i}}{\partial x_{j}}\right) \quad \text { and } \quad \xi(x, t)=\frac{b(x, t)}{|b(x, t)|} .
$$

In terms of the particle trajectory mapping we can rewrite (1.11) as

$$
\frac{\partial}{\partial t}|b(X(a, t), t)|=\alpha(X(a, t), t)|b(X(a, t), t)| .
$$

Integrating (1.12) along the particle trajectories $\{X(a, t)\}$, we have

$$
|b(X(a, t), t)|=\left|b_{0}(a)\right| \exp \left[\int_{0}^{t} \alpha(X(a, s), s) d s\right] .
$$


Taking into account the simple estimates

$$
-\|\nabla v(\cdot, t)\|_{L^{\infty}} \leq \alpha(x, t) \leq\|\nabla v(\cdot, t)\|_{L^{\infty}} \quad \forall x \in \mathbb{R}^{n}
$$

we obtain from (1.13) that

$$
\begin{aligned}
\left|b_{0}(a)\right| \exp \left[-\int_{0}^{t}\|\nabla v(\cdot, s)\|_{L^{\infty}} d s\right] \leq|b(X(a, t), t)| \\
\leq\left|b_{0}(a)\right| \exp \left[\int_{0}^{t}\|\nabla v(\cdot, s)\|_{L^{\infty}} d s\right]
\end{aligned}
$$

which, in terms of the back-to-label map, can be rewritten as

$$
\begin{aligned}
\left|b_{0}(A(x, t))\right| \exp [ & \left.-\int_{0}^{t}\|\nabla v(\cdot, s)\|_{L^{\infty}} d s\right] \leq|b(x, t)| \\
& \leq\left|b_{0}(A(x, t))\right| \exp \left[\int_{0}^{t}\|\nabla v(\cdot, s)\|_{L^{\infty}} d s\right] .
\end{aligned}
$$

Combining this with the self-similar representation formula in (1.9), we have

$$
\begin{aligned}
\left|b_{0}(A(x, t))\right| \exp \left[-\int_{0}^{t}\right. & \left.\|\nabla v(\cdot, s)\|_{L^{\infty}} d s\right] \leq G(t)\left|b_{0}(F(t) x)\right| \\
& \leq\left|b_{0}(A(x, t))\right| \exp \left[\int_{0}^{t}\|\nabla v(\cdot, s)\|_{L^{\infty}} d s\right] .
\end{aligned}
$$

Given $q \in\left(0, q_{1}\right)$, computing $L^{q}\left(\mathbb{R}^{n}\right)$ norm of the each side of (1.15), we derive

$$
\begin{array}{r}
\left\|b_{0}\right\|_{L^{q}} \exp \left[-\int_{0}^{t}\|\nabla v(\cdot, s)\|_{L^{\infty}} d s\right] \leq G(t)[\operatorname{det}(F(t))]^{-\frac{1}{q}}\left\|b_{0}\right\|_{L^{q}} \\
\leq\left\|b_{0}\right\|_{L^{q}} \exp \left[\int_{0}^{t}\|\nabla v(\cdot, s)\|_{L^{\infty}} d s\right]
\end{array}
$$

where we used the fact $\operatorname{det}(\nabla A(x, t)) \equiv 1$. Now, suppose $B \neq 0$, which is equivalent to assuming that $b_{0} \neq 0$, then we divide (1.16) by $\left\|b_{0}\right\|_{L^{q}}$ to obtain

$$
\begin{aligned}
& \exp \left[-\int_{0}^{t}\|\nabla v(\cdot, s)\|_{L^{\infty}} d s\right] \leq G(t)[\operatorname{det}(F(t))]^{-\frac{1}{q}} \\
& \leq \exp \left[\int_{0}^{t}\|\nabla v(\cdot, s)\|_{L^{\infty}} d s\right] .
\end{aligned}
$$


If there exists $t_{1} \in(0, T)$ such that $\operatorname{det}\left(F\left(t_{1}\right)\right) \neq 1$, then either $\operatorname{det}\left(F\left(t_{1}\right)\right)>1$ or $\operatorname{det}\left(F\left(t_{1}\right)\right)<1$. In either case, setting $t=t_{1}$ and passing $q \searrow 0$ in (1.17), we deduce that

$$
\int_{0}^{t_{1}}\|\nabla v(\cdot, s)\|_{L^{\infty}} d s=\infty .
$$

This contradicts with the assumption that the flow is smooth on $(0, T)$, i.e $v \in C^{1}\left([0, T) ; C_{0}^{1}\left(\mathbb{R}^{n}\right)\right)$, which is implied by the by the explicit representation formula (1.1)-(1.2), combined with the assumption (i). Hence we need to have $B=0$. Setting $B=0$ in the system (MHD), it reduces to the incompressible Euler system, and the vorticity $\omega$ satisfies

$$
\frac{\partial \omega}{\partial t}+(v \cdot \nabla) \omega=(\omega \cdot \nabla) v
$$

Multiplying (1.18) by $\omega$, we obtain that

$$
\frac{\partial|\omega|}{\partial t}+(v \cdot \nabla)|\omega|=\alpha|\omega|
$$

with the same $\alpha$ as in (1.11). From now on can repeat the above argument (1.12)-(1.17) word by word, and conclude that $\Omega=\operatorname{curl} V=0$ on $\mathbb{R}^{n}$. Hence, $V=\nabla h$ for some scalar function $h \in C^{2}\left(\mathbb{R}^{n}\right)$. Since we also have $0=\operatorname{div}$ $V=\Delta h$, we find that $V=\nabla h$ is harmonic in $\mathbb{R}^{n}$. Combining this with the hypothesis, $V \in C_{0}^{1}\left(\mathbb{R}^{n}\right)$, we obtain that $V=0$.

Proof of Theorem 1.1 We apply Theorem 1.2 with

$$
\Phi_{j}(t)=\left(T_{*}-t\right)^{-\frac{1}{\alpha+1}} I, \quad \text { and } \quad \Psi_{j}(t)=\left(T_{*}-t\right)^{-1}
$$

for $j=1,2$, where $I$ is the unit matrix in $\mathbb{R}^{n \times n}$. If $\alpha \neq-1$ and $t \neq 0$, then

$$
\operatorname{det}\left(\Phi_{j}(t)\right)=\left(T_{*}-t\right)^{-\frac{n}{\alpha+1}} \neq T_{*}^{-\frac{n}{\alpha+1}}=\operatorname{det}\left(\Phi_{j}(0)\right)
$$

for each $j=1,2$. Hence, we conclude that $B=V=0$ by Theorem 1.2 .

\section{Asymptotically self-similar singularities}

In this section we consider scenario of more refined possibility of 'asymptotically self-similar singularity', which means that the local in time smooth 
solution evolves into a self-similar profile as the possible singularity time is approached. The meaning of it will be more clear in the statements of Theorem 2.2. A similar notion was considered previously by Giga and Kohn in the context of the nonlinear scalar heat equation in [13]. Recently, the author of this paper ([5]) considered similar notion in the context of 3D Euler and the 3D Navier-Stokes equations(see also [15]), and was excluded under suitable conditions on the profile. We apply similar methods used in [5] for our case of (MHD). In the proof in 5] we crucially use a new type of continuation principle of the local solutions of 3D Euler equations, where the use of critical homogeneous Besov space $\dot{B}_{\infty, 1}^{0}\left(\mathbb{R}^{n}\right)$ (see subsection of preliminaries below for the definition) was essential. For our proof we need to establish another continuation principle for our local solutions of (MHD), which is Theorem 2.1. For such continuation principle we use different critical Besov

space, $\dot{B}_{2,1}^{\frac{n}{2}}\left(\mathbb{R}^{n}\right)$, which is technically important in order to handle the more complicated nonlinear structure in (MHD) than the case of the Euler system.

\subsection{Preliminaries}

We follow [22] (see also [7]). Let $\mathcal{S}$ be the Schwartz class of rapidly decreasing functions. Given $f \in \mathcal{S}$, its Fourier transform $\mathcal{F}(f)=\hat{f}$ is defined by

$$
\hat{f}(\xi)=\frac{1}{(2 \pi)^{n / 2}} \int e^{-i x \cdot \xi} f(x) d x .
$$

We consider $\varphi \in \mathcal{S}$ satisfying $\operatorname{Supp} \hat{\varphi} \subset\left\{\xi \in \mathbb{R}^{n}\left|\frac{1}{2} \leq\right| \xi \mid \leq 2\right\}$, and $\hat{\varphi}(\xi)>0$ if $\frac{1}{2}<|\xi|<2$. Setting $\hat{\varphi}_{j}=\hat{\varphi}\left(2^{-j} \xi\right.$ ) (In other words, $\varphi_{j}(x)=2^{j n} \varphi\left(2^{j} x\right)$.), we can adjust the normalization constant in front of $\hat{\varphi}$ so that

$$
\sum_{j \in \mathbb{Z}} \hat{\varphi}_{j}(\xi)=1 \quad \forall \xi \in \mathbb{R}^{n} \backslash\{0\}
$$

Given $k \in \mathbb{Z}$, we define the function $S_{k} \in \mathcal{S}$ by its Fourier transform

$$
\hat{S}_{k}(\xi)=1-\sum_{j \geq k+1} \hat{\varphi}_{j}(\xi)
$$

We observe

$$
\operatorname{Supp} \hat{\varphi}_{j} \cap \operatorname{Supp} \hat{\varphi}_{j^{\prime}}=\emptyset \text { if }\left|j-j^{\prime}\right| \geq 2 \text {. }
$$


Let $s \in \mathbb{R}, p, q \in[0, \infty]$. Given $f \in \mathcal{S}^{\prime}$, we denote $\Delta_{j} f=\varphi_{j} * f$. Then the homogeneous Besov semi-norm $\|f\|_{\dot{B}_{p, q}^{s}}$ is defined by

$$
\|f\|_{\dot{B}_{p, q}^{s}}=\left\{\begin{array}{l}
{\left[\sum_{-\infty}^{\infty} 2^{j q s}\left\|\Delta_{j} f\right\|_{L^{p}}^{q}\right]^{\frac{1}{q}} \text { if } q \in[1, \infty)} \\
\sup _{j}\left[2^{j s}\left\|\Delta_{j} f\right\|_{L^{p}}\right] \text { if } q=\infty
\end{array}\right.
$$

The homogeneous Besov space $\dot{B}_{p, q}^{s}$ is a quasi-normed space with the quasinorm given by $\|\cdot\|_{\dot{B}_{p, q}^{s}}$. The norm $\|\cdot\|_{\dot{B}_{p, q}^{s}}$ is actually defined up to addition of polynomials(namely, if $f_{1}-f_{2}$ is a polynomial, then both of $f_{1}$ and $f_{2}$ give the same norm), and the space $\dot{B}_{p, q}^{s}\left(\mathbb{R}^{n}\right)$ is defined as the quotient space of a class of functions with finite norm, $\|\cdot\|_{\dot{B}_{s, q}^{s}}$, divided by the space of polynomials in $\mathbb{R}^{n}$. For $s>0$ the inhomogeneous Besov space norm $\|f\|_{B_{p, q}^{s}}$ of $f \in \mathcal{S}^{\prime}$ is defined as $\|f\|_{B_{p, q}^{s}}=\|f\|_{L^{p}}+\|f\|_{\dot{B}_{p, q}^{s}}$. Let us now state some basic properties for the Besov spaces.

(i) Bernstein's Lemma: Assume that $f \in L^{p}, 1 \leq p \leq \infty$, and supp $\hat{f} \subset$ $\left\{2^{j-2} \leq|\xi|<2^{j}\right\}$, then there exists a constant $C_{k}$ such that the following inequality holds:

$$
C_{k}^{-1} 2^{j k}\|f\|_{L^{p}} \leq\left\|D^{k} f\right\|_{L^{p}} \leq C_{k} 2^{j k}\|f\|_{L^{p}} .
$$

(ii) We have the equivalence of norms

$$
\left\|D^{k} f\right\|_{\dot{B}_{p, q}^{s}} \sim\|f\|_{\dot{B}_{p, q}^{s+k}} .
$$

(iii) If $s$ satisfies $s \in\left(-\frac{n}{p}-1, \frac{n}{p}\right]$, then we have

$$
\left\|\left[u, \Delta_{j}\right] w\right\|_{L^{p}} \leq c_{j} 2^{-j(s+1)}\|u\|_{\dot{B}_{p, 1}^{\frac{n}{p}+1}}\|w\|_{\dot{B}_{p, 1}^{s}}
$$

with $\sum_{j \in \mathbb{Z}} c_{j} \leq 1$. In the above, we denote

$$
\left[u, \Delta_{j}\right] w=u \Delta_{j} w-\Delta_{j}(u w) .
$$

(iv) We have the following embedding relations for $1 \leq p<\infty$ :

$$
\dot{B}_{p, 1}^{\frac{n}{p}}\left(\mathbb{R}^{n}\right) \hookrightarrow C_{0}\left(\mathbb{R}^{n}\right) .
$$

In the above (i) and (ii) are standard(see e.g. [22, 7] for the proofs). The commutator estimate (iii) is proved in [11], and (iv) is proved in [2]. 


\subsection{Statement of the theorems}

In order to prove our main theorem on the asymptotically self-similar singularities we first establish the following continuation principle for local classical solution of (MHD), which is interesting in itself.

Theorem 2.1 Let $p \in[1, \infty)$ and $(v, b) \in\left[C\left([0, T) ; H^{m}\left(\mathbb{R}^{n}\right)\right)\right]^{2}, m>n / 2+$ 1 , be a classical solution to (MHD). There exists an absolute constant $\eta>0$ such that if

$$
\inf _{0 \leq t<T}(T-t)\left\{\|\omega(t)\|_{\dot{B}_{2,1}^{\frac{n}{2}}}+\|j(t)\|_{\dot{B}_{2,1}^{\frac{n}{2}}}\right\}<\eta,
$$

where $\omega=$ curl $v, j=$ curl $b$, then $(v(x, t), b(x, t))$ can be extended to a solution of $(M H D)$ in $[0, T+\delta] \times \mathbb{R}^{n}$, and belongs to $C\left([0, T+\delta] ; H^{m}\left(\mathbb{R}^{n}\right)\right)$ for some $\delta>0$.

Remark 2.1 The proof of the local existence of solutions to (MHD) for $v_{0}, b_{0} \in$ $H^{m}\left(\mathbb{R}^{n}\right), m>n / 2+1$, is standard, adapting for example the proof of local existence theorem for the Euler equations in [16](see also [19]). The above theorem says that if $T_{*}$ is the first time of singularity, then we have the lower estimate of the blow-up rate; there exists $t_{0} \in\left[0, T_{*}\right)$ such that

$$
\|\omega(t)\|_{\dot{B}_{2,1}^{\frac{n}{2}}}+\|j(t)\|_{\dot{B}_{2,1}^{\frac{n}{2}}} \geq \frac{C}{T_{*}-t} \quad \forall t \in\left(t_{0}, T_{*}\right) .
$$

As a consequence of the above theorem we can exclude easily the possibility of 'small' self-similar singularities. The proof is in the next subsection.

Corollary 2.1 Let $(v, b)$ be a classical solution to (MHD). Suppose there exist $\eta>0, T>0$ and $t_{0} \in(0, T)$ such that we have representation

$$
\begin{aligned}
& v(x, t)=\frac{1}{(T-t)^{\frac{\alpha}{\alpha+1}}} \bar{V}\left(\frac{x}{(T-t)^{\frac{1}{\alpha+1}}}\right) \quad \forall(x, t) \in \mathbb{R}^{n} \times\left(t_{0}, T\right), \\
& b(x, t)=\frac{1}{(T-t)^{\frac{\alpha}{\alpha+1}}} \bar{B}\left(\frac{x}{(T-t)^{\frac{1}{\alpha+1}}}\right) \quad \forall(x, t) \in \mathbb{R}^{n} \times\left(t_{0}, T\right),
\end{aligned}
$$

where $(\bar{V}, \bar{B}) \in\left[H^{m}\left(\mathbb{R}^{n}\right)\right]^{2}, m>n / 2+1$, and $\bar{\Omega}=$ curl $\bar{V}, \bar{J}=$ curl $\bar{B}$ satisfy

$$
\|\bar{\Omega}\|_{\dot{B}_{2,1}^{\frac{n}{2}}}+\|\bar{J}\|_{\dot{B}_{2,1}^{\frac{n}{2}}}<\eta \text {. }
$$

Then, $\bar{V}=\bar{B}=0$. 
In particular we note that we have milder decay condition at infinity for $\bar{\Omega}$ in the above corollary than in Theorem 1.1.

The following theorem exclude the possibility of a type of asymptotically self-similar singularity for (MHD).

Theorem 2.2 Let $(v, b) \in\left[C\left([0, T) ; H^{m}\left(\mathbb{R}^{n}\right)\right)\right]^{2}, m>n / 2+1$, be a classical solutions to (MHD). Suppose there exist functions $\bar{V}, \bar{B}$ satisfying the condition (ii) for $V, B$ in Theorem 1.1 such that the following boundedness and the convergence hold true:

$$
\begin{aligned}
& \sup _{0<t<T}(T-t)^{\frac{\alpha-n}{\alpha+1}}\left\|v(\cdot, t)-\frac{1}{(T-t)^{\frac{\alpha}{\alpha+1}}} \bar{V}\left(\frac{\cdot}{(T-t)^{\frac{1}{\alpha+1}}}\right)\right\|_{L^{1}} \\
& \quad+\sup _{0<t<T}(T-t)^{\frac{\alpha-n}{\alpha+1}}\left\|b(\cdot, t)-\frac{1}{(T-t)^{\frac{\alpha}{\alpha+1}}} \bar{B}\left(\frac{\cdot}{(T-t)^{\frac{1}{\alpha+1}}}\right)\right\|_{L^{1}}<\infty,
\end{aligned}
$$

and

$$
\begin{aligned}
& \lim _{t \nearrow T}(T-t)\left\|\omega(\cdot, t)-\frac{1}{T-t} \bar{\Omega}\left(\frac{\cdot}{(T-t)^{\frac{1}{\alpha+1}}}\right)\right\|_{\dot{B}_{2,1}^{\frac{n}{2}}} \\
& \quad+\lim _{t \nearrow T}(T-t)\left\|j(\cdot, t)-\frac{1}{T-t} \bar{J}\left(\frac{\cdot}{(T-t)^{\frac{1}{\alpha+1}}}\right)\right\|_{\dot{B}_{2,1}^{\frac{n}{2}}}=0,
\end{aligned}
$$

where $\bar{\Omega}=\operatorname{curl} \bar{V}$ and $\bar{J}=\operatorname{curl} \bar{B}$. Then, $\bar{V}=\bar{B}=0$, and $(v, b)$ can be extended to a solution of $(M H D)$ in $[0, T+\delta] \times \mathbb{R}^{n}$, and belongs to $C\left([0, T+\delta] ; H^{m}\left(\mathbb{R}^{n}\right)\right)$ for some $\delta>0$.

Remark 2.3 We note that we did not impose the condition (i) for $\bar{V}$ and $\bar{B}$. This condition is guaranteed by the assumption of (2.5) and (2.6)

Remark 2.4 We note that Theorem 1.2 still does not exclude the possibility that the vorticity and the magnetic field converge to the asymptotically self-similar singularity in the weaker sense than $L^{\infty}$ sense. Namely, a selfsimilar vorticity profile could be approached from a local classical solution 
in the pointwise sense in space, or in the $L^{q}\left(\mathbb{R}^{n}\right)$ sense for some $q \in(1, \infty)$.

As an immediate corollary of Theorem 2.2 we have the following information of the behaviors of solution near possible singularity, which is not necessarily of the self-similar type.

Corollary 2.2 Let $(v, b) \in\left[C\left(\left[0, T_{*}\right) ; H^{m}\left(\mathbb{R}^{n}\right)\right)\right]^{2}, m>n / 2+1$, be a classical solutions to (MHD), which blows up at $T_{*}$. We expand the solution of the form:

$$
\begin{aligned}
& v(x, t)=\frac{1}{\left(T_{*}-t\right)^{\frac{\alpha}{\alpha+1}}} \bar{V}\left(\frac{x}{\left(T_{*}-t\right)^{\frac{1}{\alpha+1}}}\right)+\bar{v}(x, t), \\
& b(x, t)=\frac{1}{\left(T_{*}-t\right)^{\frac{\alpha}{\alpha+1}}} \bar{B}\left(\frac{x}{\left(T_{*}-t\right)^{\frac{1}{\alpha+1}}}\right)+\bar{b}(x, t),
\end{aligned}
$$

where $(\bar{V}, \bar{B})$ satisfies the conditions (i)-(ii) for $(V, B)$ in Theorem 1.1, and $\alpha \neq-1, t \in\left[0, T_{*}\right)$. Then, either

$$
\lim \sup _{t \nearrow T_{*}}\left(T_{*}-t\right)^{\frac{\alpha-n}{\alpha+1}}\|\bar{v}(\cdot, t)\|_{L^{1}}=\infty
$$

or there exists $\varepsilon_{0}>0$ such that

$$
\lim \sup _{t \nearrow T_{*}}\left(T_{*}-t\right)\left(\|\bar{\omega}(t)\|_{\dot{B}_{2,1}^{\frac{n}{2}}}+\|\bar{j}(t)\|_{\dot{B}_{2,1}^{\frac{n}{2}}}\right)>\varepsilon_{0},
$$

where $\bar{\omega}=\operatorname{curl} \bar{v}$, and $\bar{j}=$ curl $\bar{b}$ respectively.

\subsection{Proof of the theorems}

Proof of Theorem 2.1 We set

$$
v_{ \pm}=v \pm b, \quad \pi=p+\frac{1}{2}|b|^{2} .
$$

Adding and subtracting the first and the second equations of (MHD), we obtain

$$
\frac{\partial v_{ \pm}}{\partial t}+\left(v_{\mp} \cdot \nabla\right) v_{ \pm}=-\nabla \pi
$$


Taking the operation $\Delta_{j}$ on (2.11), we have

$$
\frac{\partial}{\partial t} \Delta_{j} v_{ \pm}+\left(v_{\mp} \cdot \nabla\right) \Delta_{j} v_{ \pm}=\left[v_{\mp}, \Delta_{j}\right] \cdot \nabla v_{ \pm}+\nabla\left(\Delta_{j} \pi\right) .
$$

Multiplying $\Delta_{j} v_{ \pm}$on the both sides of (2.12) and integrating over $\mathbb{R}^{n}$, we obtain, after integration by part,

$$
\frac{1}{2} \frac{d}{d t}\left\|\Delta_{j} v_{ \pm}\right\|_{L^{2}}^{2} \leq\left\|\left[\Delta_{j}, v_{\mp}\right] \cdot \nabla v_{ \pm}\right\|_{L^{2}}\left\|\Delta_{j} v_{ \pm}\right\|_{L^{2}} .
$$

Dividing the both sides of (2.13) by $\left\|\Delta_{j} v_{ \pm}\right\|_{L^{2}}$, and using the commutator estimates (2.1), we deduce

$$
\frac{d}{d t}\left\|\Delta_{j} v_{ \pm}\right\|_{L^{2}} \leq C c_{j} 2^{-\left(\frac{n}{2}+1\right) j}\left(\left\|v_{ \pm}\right\|_{\dot{B}_{2,1}^{\frac{n}{2}+1}}+\left\|v_{\mp}\right\|_{\dot{B}_{2,1}^{\frac{n}{2}+1}}\right)\left\|v_{ \pm}\right\|_{\dot{B}_{2,1}^{\frac{n}{2}+1}} .
$$

Multiplying $2^{\left(\frac{n}{2}+1\right) j}$ on the both sides of (2.14), and taking summation over $j \in \mathbb{Z}$, it reduces that

$$
\frac{d}{d t}\left\|v_{ \pm}(t)\right\|_{\dot{B}_{2,1}^{\frac{n}{2}+1}} \leq C\left(\left\|v_{ \pm}(t)\right\|_{\dot{B}_{2,1}^{\frac{n}{2}+1}}+\left\|v_{\mp}(t)\right\|_{\dot{B}_{2,1}^{\frac{n}{2}+1}}\right)\left\|v_{ \pm}(t)\right\|_{\dot{B}_{2,1}^{\frac{n}{2}+1}} .
$$

Let us define

$$
X(t)=\left\|v_{+}(t)\right\|_{\dot{B}_{2,1}^{\frac{n}{2}+1}}+\left\|v_{-}(t)\right\|_{\dot{B}_{2,1}^{\frac{n}{2}+1}} .
$$

Then, from (2.15) we have

$$
\frac{d}{d t} X(t) \leq C X(t)^{2}
$$

By the Gronwall lemma we deduce

$$
X(t) \leq \frac{X(0)}{1-C t X(0)} .
$$

Translating in time, we have instead of (2.17)

$$
X\left(T_{1}\right) \leq \frac{C_{1} X(t)}{1-C_{2}\left(T_{1}-t\right) X(t)}
$$

for all $T_{1} \in(0, T)$ and $t \in\left[0, T_{1}\right)$, where $C_{1}, C_{2}$ are absolute constants. We set $\eta=\frac{1}{2 C_{2}}$. For such $\eta$, we suppose (2.3) holds true. Then, there exists 
$t_{1} \in[0, T)$ such that $\left(T-t_{1}\right) X\left(t_{1}\right)<\eta$. Fixing $t=t_{1}$, and passing $T_{1} \nearrow T$ in (2.18), we find that

$$
\lim \sup _{T_{1} \nearrow T} X\left(T_{1}\right) \leq 2 C_{1} X\left(t_{1}\right)<\infty,
$$

and hence, we obtain the estimate for the homogeneous Besov norm,

$$
\sup _{0<t<T}\left(\|v(t)\|_{\dot{B}_{2,1}^{\frac{n}{2}+1}}+\|b(t)\|_{\dot{B}_{2,1}^{\frac{n}{2}+1}}\right)<C \sup _{0<t<T} X(t):=M(T)<\infty .
$$

if our hypothesis (2.3) holds true. Therefore, we have

$$
\begin{gathered}
\int_{0}^{T}\left\{\|\omega(t)\|_{L^{\infty}}+\|j(t)\|_{L^{\infty}}\right\} d t \leq \int_{0}^{T}\left\{\|\nabla v(t)\|_{L^{\infty}}+\|\nabla b(t)\|_{L^{\infty}}\right\} d t \\
\leq C \int_{0}^{T}\left\{\|v(t)\|_{\dot{B}_{2,1}^{\frac{n}{2}+1}}+\|b(t)\|_{\dot{B}_{2,1}^{\frac{n}{2}+1}}\right\} d t \leq C M(T) T<\infty,
\end{gathered}
$$

where we used the embedding $\dot{B}_{2,1}^{\frac{n}{2}}\left(\mathbb{R}^{n}\right) \hookrightarrow L^{\infty}\left(\mathbb{R}^{n}\right)$. Applying the BKM type of blow-up criterion for (MHD) derived in [3], we can continue our local solution $(v(x, t), b(x, t))$ until $t=T+\delta$, and $(v, b) \in\left[C\left([0, T+\delta] ; H^{m}\left(\mathbb{R}^{n}\right)\right)\right]^{2}$ for some $\delta>0$, where $m>n / 2+1$.

Proof of Corollary 2.1 We just observe that

$$
(T-t)\|\omega(t)\|_{\dot{B}_{2,1}^{\frac{n}{2}}}=\|\bar{\Omega}\|_{\dot{B}_{2,1}^{\frac{n}{2}},}, \quad(T-t)\|j(t)\|_{\dot{B}_{2,1}^{\frac{n}{2}}}=\|\bar{J}\|_{\dot{B}_{2,1}^{\frac{n}{2}}}
$$

for all $t \in\left(t_{0}, T\right)$. Hence, our smallness condition, $\|\bar{\Omega}\|_{\dot{B}_{2,1}^{\frac{n}{2}}}+\|\bar{J}\|_{\dot{B}_{2,1}^{\frac{n}{2}}}<\eta$, leads to

$$
\inf _{t_{0}<t<T}(T-t)\left\{\|\omega(t)\|_{\dot{B}_{2,1}^{\frac{n}{2}}}+\|j(t)\|_{\dot{B}_{2,1}^{\frac{n}{2}}}\right\}<\eta .
$$

Applying Theorem 2.1, for initial time at $t=t_{0}$, we conclude that $(v, b) \in$ $\left[C^{1}\left(\left[t_{0}, T\right) ; H^{m}\left(\mathbb{R}^{n}\right)\right)\right]^{2}$ cannot have singularity at $t=t_{0}$, hence we need to have $V=B=0$.

Proof of Theorem 2.2 We change variables from the physical ones $(x, t) \in$ $\mathbb{R}^{n} \times[0, T)$ to the 'self-similar variables' $(y, s) \in \mathbb{R}^{n} \times[0, \infty)$ as follows:

$$
y=\frac{x}{(T-t)^{\frac{1}{\alpha+1}}}, \quad s=\frac{1}{\alpha+1} \log \left(\frac{T}{T-t}\right) .
$$


Based on this change of variables, we transform the functions $(v, p) \mapsto(V, P)$ according to

$$
\begin{aligned}
v(x, t) & =\frac{1}{(T-t)^{\frac{\alpha}{\alpha+1}}} V(y, s), \\
b(x, t) & =\frac{1}{(T-t)^{\frac{\alpha}{\alpha+1}}} B(y, s), \\
p(x, t) & =\frac{1}{(T-t)^{\frac{2 \alpha}{\alpha+1}}} P(y, s) .
\end{aligned}
$$

Substituting $(v, b, p)$ into the $(M H D)$, we obtain the following equivalent evolution equations for $(V, P)$,

$$
\left\{\begin{array}{l}
\frac{1}{\alpha+1} V_{s}+\frac{\alpha}{\alpha+1} V+\frac{1}{\alpha+1}(y \cdot \nabla) V+(V \cdot \nabla) V=(B \cdot \nabla) B-\nabla\left(P+\frac{1}{2}|V|^{2}\right), \\
\frac{1}{\alpha+1} B_{s}+\frac{\alpha}{\alpha+1} B+\frac{1}{\alpha+1}(y \cdot \nabla) B+(V \cdot \nabla) B=(B \cdot \nabla) V, \\
\quad \operatorname{div} V=\operatorname{div} B=0, \\
V(y, 0)=V_{0}(y)=T^{\frac{\alpha}{\alpha+1}} v_{0}\left(T^{\frac{1}{\alpha+1}} y\right), \quad B(y, 0)=B_{0}(y)=T^{\frac{\alpha}{\alpha+1}} b_{0}\left(T^{\frac{1}{\alpha+1}} y\right) .
\end{array}\right.
$$

In terms of $(V, B)$ the conditions $(\underline{2.5})$ and $(\underline{2.6})$ are translated into

$$
\sup _{0<s<\infty}\|V(\cdot, s)-\bar{V}(\cdot)\|_{L^{1}}+\sup _{0<s<\infty}\|B(\cdot, s)-\bar{B}(\cdot)\|_{L^{1}}<\infty
$$

and

$$
\lim _{s \rightarrow \infty}\|\Omega(\cdot, s)-\bar{\Omega}(\cdot)\|_{\dot{B}_{2,1}^{\frac{n}{2}}}=\lim _{s \rightarrow \infty}\|J(\cdot, s)-\bar{J}(\cdot)\|_{\dot{B}_{2,1}^{\frac{n}{2}}}=0 .
$$

From the fact that the Calderon-Zygmund singular integral operator maps $\dot{B}_{2,1}^{\frac{n}{2}}\left(\mathbb{R}^{n}\right)$ into itself boundedly, we obtain from (2.25)

$$
\lim _{s \rightarrow \infty}\|V(\cdot, s)-\bar{V}(\cdot)\|_{\dot{B}_{2,1}^{\frac{n}{2}+1}}=\lim _{s \rightarrow \infty}\|B(\cdot, s)-\bar{B}(\cdot)\|_{\dot{B}_{2,1}^{\frac{n}{2}+1}}=0,
$$

from which, thanks to the embedding (2.2) and (2.24), we have

$$
\lim _{s \rightarrow \infty}\|V(\cdot, s)-\bar{V}(\cdot)\|_{C^{1}\left(B_{R}\right)}=\lim _{s \rightarrow \infty}\|B(\cdot, s)-\bar{B}(\cdot)\|_{C^{1}\left(B_{R}\right)}=0
$$

for all $R>0$, where $B_{R}=\left\{x \in \mathbb{R}^{n}|| x \mid<R\right\}$. Moreover, we find that $\bar{V}, \bar{B} \in C_{0}\left(\mathbb{R}^{n}\right)$, satisfying the condition (i) of Theorem 1.1. Similarly to [15], 
we consider scalar test functions $\xi \in C_{c}^{1}(0,1)$ with $\int_{0}^{1} \xi(s) d s \neq 0, \psi \in C_{c}^{1}\left(\mathbb{R}^{n}\right)$ and the vector test function $\phi=\left(\phi_{1}, \cdots, \phi_{n}\right) \in C_{c}^{1}\left(\mathbb{R}^{n}\right)$ with $\operatorname{div} \phi=0$. We multiply the first equation of $(2.23)$ by $\xi(s-k) \phi(y)$, and integrate it over $\mathbb{R}^{n} \times[k, k+1]$, and then we integrate by part for the terms including the time derivative and the pressure term to obtain

$$
\begin{aligned}
& -\int_{0}^{1} \int_{\mathbb{R}^{n}} \xi_{s}(s) \phi(y) \cdot V(y, s+k) d y d s \\
& +\int_{0}^{1} \int_{\mathbb{R}^{n}} \xi(s) \phi(y) \cdot[\alpha V+(y \cdot \nabla) V+(\alpha+1)(V \cdot \nabla) V](y, s+k) d y d s \\
& \left.\quad-(\alpha+1) \int_{0}^{1} \int_{\mathbb{R}^{n}} \xi(s) \phi(y) \cdot(B \cdot \nabla) B\right](y, s+k) d y d s=0
\end{aligned}
$$

and

$$
\begin{aligned}
& -\int_{0}^{1} \int_{\mathbb{R}^{n}} \xi_{s}(s) \psi(y) B(y, s+k) d y d s \\
& +\int_{0}^{1} \int_{\mathbb{R}^{n}} \xi(s) \psi(y)[\alpha B+(y \cdot \nabla) B+(\alpha+1)(V \cdot \nabla) B](y, s+k) d y d s \\
& \left.\quad-(\alpha+1) \int_{0}^{1} \int_{\mathbb{R}^{n}} \xi(s) \psi(y)(B \cdot \nabla) V\right](y, s+k) d y d s=0
\end{aligned}
$$

Passing to the limit $k \rightarrow \infty$ in (2.28)-(2.29), using the convergence (2.27), $\int_{0}^{1} \xi_{s}(s) d s=0$ and $\int_{0}^{1} \xi(s) d s \neq 0$, we find that $\bar{V}, \bar{B} \in C_{0}^{1}\left(\mathbb{R}^{n}\right)$ satisfies

$$
\int_{\mathbb{R}^{n}}[\alpha \bar{V}+(y \cdot \nabla) \bar{V}+(\alpha+1)(\bar{V} \cdot \nabla) \bar{V}-(\alpha+1)(\bar{B} \cdot \nabla) \bar{B}] \cdot \phi d y=0,
$$

and

$$
\int_{\mathbb{R}^{n}}[\alpha \bar{B}+(y \cdot \nabla) \bar{B}+(\alpha+1)(\bar{V} \cdot \nabla) \bar{B}-(\alpha+1)(\bar{B} \cdot \nabla) \bar{V}] \psi d y=0,
$$

for all vector test function $\phi \in C_{c}^{1}\left(\mathbb{R}^{n}\right)$ with div $\phi=0$, and scalar test function $\psi \in C_{c}^{1}\left(\mathbb{R}^{n}\right)$ Hence, there exists a scalar function $\bar{P}^{\prime}$, which can be written without loss of generality that $\bar{P}^{\prime}=\bar{P}+\frac{1}{2}|\bar{B}|^{2}$ for another scalar function $\bar{P}$, such that

$$
\begin{aligned}
\alpha \bar{V}+(y \cdot \nabla) \bar{V}+(\alpha+1)(\bar{V} \cdot \nabla) \bar{V}=( & \alpha+1)(\bar{B} \cdot \nabla) \bar{B} \\
& -(\alpha+1) \nabla\left(\bar{P}+\frac{1}{2}|\bar{B}|^{2}\right)
\end{aligned}
$$


and

$$
\alpha \bar{B}+(y \cdot \nabla) \bar{B}+(\alpha+1)(\bar{V} \cdot \nabla) \bar{B}=(\alpha+1)(\bar{B} \cdot \nabla) \bar{V}
$$

On the other hand, we can pass $s \rightarrow \infty$ directly in the incompressibility equations for $V$ and $B$ in (2.23) to have

$$
\operatorname{div} \bar{V}=\operatorname{div} \bar{B}=0 .
$$

The equations (2.30)-(2.32) show that $(\bar{V}, \bar{B})$ is a classical solution of (1.4). Since, by hypothesis, curl $\bar{V}=\bar{\Omega}$ and $\bar{B}$ satisfy the condition (ii) of Theorem 1.1, we can deduce $\bar{V}=\bar{B}=0$ by that theorem. Hence, (2.25) leads to

$$
\lim _{s \rightarrow \infty}\|\Omega(s)\|_{\dot{B}_{2,1}^{\frac{n}{2}}}=\lim _{s \rightarrow \infty}\|J(s)\|_{\dot{B}_{2,1}^{\frac{n}{2}}}=0 .
$$

Thus, for $\eta>0$ given in Theorem 1.1 , there exists $s_{1}>0$ such that

$$
\left\|\Omega\left(s_{1}\right)\right\|_{\dot{B}_{2,1}^{\frac{n}{2}}}+\left\|J\left(s_{1}\right)\right\|_{\dot{B}_{2,1}^{\frac{n}{2}}}<\eta .
$$

Let us set $t_{1}=T\left[1-e^{(\alpha+1) s_{1}}\right]$. Going back to the original physical variables, we have

$$
\left(T-t_{1}\right)\left\|\omega\left(t_{1}\right)\right\|_{\dot{B}_{2,1}^{\frac{n}{2}}}+\left(T-t_{1}\right)\left\|j\left(t_{1}\right)\right\|_{\dot{B}_{2,1}^{\frac{n}{2}}}<\eta .
$$

Applying Theorem 2.1, we conclude the proof.

\section{The 2D magnetohydrodynamics}

In the 2D magnetohydrodynamics, namely (MHD) with $n=2$, we have the following reduced form of (MHD), using the stream function for the magnetic field, $b=\nabla^{\perp} \varphi$.

$$
(2 \mathrm{D}-\mathrm{MHD})\left\{\begin{array}{l}
\frac{\partial v}{\partial t}+(v \cdot \nabla) v=(b \cdot \nabla) b-\nabla\left(p+\frac{1}{2}|b|^{2}\right) \\
\frac{\partial \varphi}{\partial t}+(v \cdot \nabla) \varphi=0 \\
\operatorname{div} v=0, \quad b=\nabla^{\perp} \varphi \\
\varphi(x, 0)=\varphi_{0}(x), \quad v(x, 0)=v_{0}(x)
\end{array}\right.
$$

Unlike the case of 2D Euler equations, the question of finite time blowup/global regularity question in the magnetohydrodynamics is wide open 
even for the 2D case. Similar situation is for the question of global existence of weak solutions. We mention the result of global existence of weak solutions for a partial viscosity case obtained by Kozono in [17]. There are studies on the possible scenarios of finite time singularity other than that of self-similar type in [9, 10]. We note that the equation for $\varphi$ in (2D-MHD) is nothing but a divergence free transport equation, for which the nonexistence of selfsimilar singularity is obtained in [4] in a general setting. Applying the general theorem we first have the following improved version of Theorem 1.2.

Theorem 3.1 Suppose there exists $T>0$ such that we have a representation of the solution $(v, b)$ to the system (2D-MHD) with $b=\nabla^{\perp} \varphi$ such that we have the representation:

$$
\varphi(x, t)=\Psi(t) \Theta(\Phi(t) x)
$$

for all $t \in[0, T)$, where $\Psi(\cdot) \in C([0, T) ;(0, \infty)), \Phi(\cdot) \in C\left([0, T) ; \mathbb{R}^{2 \times 2}\right)$ with $\operatorname{det}(\Phi(t)) \neq 0$ on $[0, T)$. Suppose also that there exist $q_{1}<q_{2}$ with $q_{1}, q_{2} \in$ $(0, \infty]$ such that $\Theta \in L^{q_{1}}\left(\mathbb{R}^{2}\right) \cap L^{q_{2}}\left(\mathbb{R}^{2}\right)$, then either $\operatorname{det}(\Phi(t))=\operatorname{det}(\Phi(0))$ or $\Theta=0$.

The proof follows immediately by Theorem 2.2 in [4] to the first equation of (2D-MHD). Note that we do not assume any integrability condition for the vorticity in the above theorem. As a corollary of the above theorem we obtain:

Theorem 3.2 Suppose there exists $T_{*}>0$ such that we have a solution $(v, b)$ with $b=\nabla^{\perp} \varphi$ to the system (2D-MHD) with the representation:

$$
\begin{aligned}
& v(x, t)=\frac{1}{\left(T_{*}-t\right)^{\frac{\alpha}{\alpha+1}}} V\left(\frac{x}{\left(T_{*}-t\right)^{\frac{1}{\alpha+1}}}\right) \\
& \varphi(x, t)=\frac{1}{\left(T_{*}-t\right)^{\frac{\alpha-1}{\alpha+1}}} \Theta\left(\frac{x}{\left(T_{*}-t\right)^{\frac{1}{\alpha+1}}}\right)
\end{aligned}
$$

for $t \in\left(0, T_{*}\right)$ with $\alpha \neq-1$, and let $V \in H^{m}\left(\mathbb{R}^{2}\right), m>2$, and $\Theta \in L^{q_{1}}\left(\mathbb{R}^{2}\right) \cap$ $L^{q_{2}}\left(\mathbb{R}^{2}\right)$ for some $q_{1}, q_{2} \in(0, \infty]$ with $q_{1}<q_{2}$. Then, $\Theta=0$, and $V=0$.

Remark 3.1 We note that the integrability condition on the vorticity $\Omega=$ curl $V \in H^{m-1}\left(\mathbb{R}^{2}\right), m>2$, and the corresponding decay at infinity are weaker 
here than in Theorem 1.1. The assumption for $\Theta$ in the above theorem is satisfied if

$$
B=\nabla^{\perp} \Theta \in W^{-1, q_{1}}\left(\mathbb{R}^{2}\right) \cap W^{-1, q_{2}}\left(\mathbb{R}^{2}\right) \quad \forall 1<q_{1}<q_{2} \leq \infty .
$$

Since $L^{\frac{2 q}{2-q}}\left(\mathbb{R}^{2}\right) \hookrightarrow W^{-1, q}\left(\mathbb{R}^{2}\right)$ for $q \in(1,2)$, we have that the representation (1.1)-(1.2) for the solution $(v, b)$ of (MHD) for $n=2$ with $B$ satisfying

$$
B \in L^{p_{1}}\left(\mathbb{R}^{2}\right) \cap L^{p_{2}}\left(\mathbb{R}^{2}\right), \quad \forall 2<p_{1}<p_{2}<\infty
$$

implies $V=B=0$.

Proof of Theorem 3.2 Applying Theorem 3.1 to the representation of $\varphi$ in (3.3), we first conclude that $\Theta=\varphi=0$. Setting $\varphi=0$ in (2D-MHD), then the system reduces to the 2D Euler equations, for which we have the global well-posedness of the classical solutions for initial data $v(t) \in H^{m}\left(\mathbb{R}^{2}\right)$, $m>2$, at $t \in\left[0, T_{*}\right)$, and hence the representation for $v$ in (3.2) is possible only when $V=0$.

Combining Theorem 3.2 with Theorem 2.2, we also immediately obtain the following theorem.

Theorem 3.3 Let $(v, b) \in\left[C\left([0, T) ; H^{m}\left(\mathbb{R}^{2}\right)\right)\right]^{2}, m>2$, be a classical solutions to (MHD) with $n=2$. Suppose there exist functions $\bar{V}, \bar{B}=\nabla^{\perp} \bar{\Theta}$ with $\bar{V}$ and $\bar{\Theta}$, satisfying the conditions of $V$ and $\Theta$ respectively in Theorem 3.2 and $\alpha \neq-1$ such that the following estimate and convergence hold true:

$$
\begin{aligned}
& \sup _{0<t<T}(T-t)^{\frac{\alpha-2}{\alpha+1}}\left\|v(\cdot, t)-\frac{1}{(T-t)^{\frac{\alpha}{\alpha+1}}} \bar{V}\left(\frac{\cdot}{(T-t)^{\frac{1}{\alpha+1}}}\right)\right\|_{L^{1}} \\
& \quad+\sup _{0<t<T}(T-t)^{\frac{\alpha-2}{\alpha+1}}\left\|b(\cdot, t)-\frac{1}{(T-t)^{\frac{\alpha}{\alpha+1}}} \bar{B}\left(\frac{\cdot}{(T-t)^{\frac{1}{\alpha+1}}}\right)\right\|_{L^{1}}<\infty,
\end{aligned}
$$


and

$$
\begin{aligned}
& \lim _{t \nearrow T}(T-t)\left\|\omega(\cdot, t)-\frac{1}{T-t} \bar{\Omega}\left(\frac{\cdot}{(T-t)^{\frac{1}{\alpha+1}}}\right)\right\|_{\dot{B}_{2,1}^{1}} \\
& \quad+\lim _{t \nearrow T}(T-t)\left\|j(\cdot, t)-\frac{1}{T-t} \bar{J}\left(\frac{\cdot}{(T-t)^{\frac{1}{\alpha+1}}}\right)\right\|_{\dot{B}_{2,1}^{1}}=0
\end{aligned}
$$

where we set

$$
\omega=\partial_{1} v_{2}-\partial_{2} v_{1}, \quad j=\partial_{1} b_{2}-\partial_{2} b_{1},
$$

and

$$
\bar{\Omega}=\partial_{1} \bar{V}_{2}-\partial_{2} \bar{V}_{1}, \quad \bar{J}=\Delta \bar{\Theta} .
$$

Then, $\bar{V}=\bar{B}=0$, and $(v, b)$ can be extended to a solution of (MHD) in $[0, T+\delta] \times \mathbb{R}^{2}$, and belongs to $C\left([0, T+\delta] ; H^{m}\left(\mathbb{R}^{2}\right)\right), m>2$, for some $\delta>0$.

\section{References}

[1] J. T. Beale, T. Kato and A. Majda, Remarks on the breakdown of smooth solutions for the 3-D Euler equations, Comm. Math. Phys., 94, (1984), pp. 61-66.

[2] G. Bourdaud, Reálisations des espaces de Besov homogènes, Ark. Math., 26, (1988), pp. 41-54.

[3] R. E. Caflisch, I. Klapper and G. Steele, Remarks on singularities, dimension and energy dissipation for ideal hydrodynamics and MHD, Comm. Math. Phys., 184, (1997), pp. 443-455.

[4] D. Chae, Nonexistence of self-similar singularities for the 3D incompressible Euler equations, Comm. Math. Phys., in press.

[5] D. Chae, Nonexistence of asymptotically self-similar singularities in the Euler and the Navier-Stokes equations, Math. Ann., in press.

[6] D. Chae, Incompressible Euler Equations: the blow-up problem and related results, ArXiv:math/0703405, to appear in Handbook on Evolutionary Differential Equations, Elsevier. 
[7] J. Y. Chemin, Perfect incompressible fluids, Clarendon Press, Oxford, (1998).

[8] P. Constantin, An Eulerian-Lagrangian approach for incompressible fluids: local theory. J. Amer. Math. Soc., 14, no. 2, (2001), pp. 263-278.

[9] D. Córdoba and C. Fefferman, Behavior of several two-dimensional fluid equations in singular scenarios, Proc. Natl. Acad. Sci. USA, 98, no. 8, (2001), pp.4311-4312.

[10] D. Córdoba and C. Marliani, Evolution of current sheets and regularity of ideal incompressible magnetic fluids in 2D, Comm. Pure Appl. Math., 53, no. 4, (2000), pp. 512-524.

[11] R. Danchin, Local theory in critical spaces for compressible viscous and heat-conductive gases, Comm. PDE, 26, (2001), pp. 1183-1233.

[12] J. D. Gibbon and K. Ohkitani, Evidence for singularity formation in a class of stretched solutions of the equations for ideal MHD, Tubes, sheets and singularities in fluid dynamics (Zakopane, 2001), Fluid Mech. Appl., 71, Kluwer Acad. Publ., Dordrecht, (2002), pp. 295-304.

[13] Y. Giga and R. V. Kohn, Asymptotically Self-Similar Blow-up of Semilinear Heat Equations, Comm. Pure Appl. Math., 38, (1985), pp. 297-319.

[14] R. Grauer,Adaptive mesh refinement for singular structures in incompressible hydro- and magnetohydrodynamic flows, Hyperbolic problems: theory, numerics, applications, I, (Zürich, 1998), Internat. Ser. Numer. Math., 129, Birkhauser, Basel, (1999), pp. 401-410.

[15] T. Y. Hou and R. Li, Nonexistence of Local Self-Similar Blowup for the 3D Incompressible Navier-Stokes Equations, arXiv-preprint, math.AP/0603126.

[16] T. Kato, Nonstationary flows of viscous and ideal fluids in $\mathbb{R}^{3}$, J. Functional Anal. 9, (1972), pp. 296-305.

[17] H. Kozono, Weak and classical solutions of the two dimensional magnetohydrodynamical equations, Tohoku Math. J., 41, (1989), pp. 471-488.

[18] J. Leray, Essai sur le mouvement d'un fluide visqueux emplissant l'espace, Acta Math. 63 (1934), pp. 193-248. 
[19] A. Majda and A. Bertozzi, Vorticity and Incompressible Flow, Cambridge Univ. Press. (2002).

[20] J. Nečas, M. Ružička and V. Šverák, On Leray's self-similar solutions of the Navier-Stokes equations, Acta Math., 176, no. 2, (1996), pp. 283-294.

[21] K. Ohkitani, $A$ note on regularity conditions on ideal magnetohydrodynamic equations, Phys. Plasmas, 13, no. 4, 044504, (2006), 3 pp.

[22] H. Triebel, Theory of Function Spaces, Birkäuser Verlag, Boston, (1983).

[23] T-P. Tsai, On Leray's self-similar solutions of the Navier-Stokes equations satisfying local energy estimates, Arch. Rat. Mech. Anal., 143, no. 1, (1998), pp. 29-51. 